\title{
AutoMedic: Fuzzy Control Development Platform for a Mobile Heart-Lung Machine
}

\author{
A. Mendoza García ${ }^{1}$, B. Baumgartner ${ }^{1}$, U. Schreiber ${ }^{2}$, M.Krane ${ }^{2}$, A.Knoll ${ }^{1}$ and R.Bauernschmitt ${ }^{2}$ \\ ${ }^{1}$ Department of Informatics, Robotics and Embedded Systems, Technische Universität München, Germany \\ ${ }^{2}$ Department of Cardiovascular Surgery, German Heart Center Munich, Technische Universität München, Germany
}

\begin{abstract}
Cardiogenic shock due to myocardial infarction is still associated with a high mortality of more than $60 \%$. Early treatment of patients having a low cardiac output with an extra-corporal circulatory support system (ECSS) could prevent multi-organ failure. Current heart-lung machines (HLM) need to be constantly supervised and operated by trained personnel, making it a difficult task to use a HLM in emergency situations. For this project we used the Lifebridge $B_{2} T$ as an existing portable, modular, rapidly available "plug and play" mechanical circulatory support system. We present the AutoMedic platform for the development of a mobile, autonomous and self-controlling ECSS. This framework was established in order to give medical doctors and engineers a common ground for the design of a controller to automate a ECSS. Such a controller has to be capable of adapting to the individual needs of each patient. Besides, such a system needs to be robust, reliable and must constantly guarantee patient safty.
\end{abstract}

Keywords-Heart-Lung Machine, Monitoring System, Fuzzy Controller, Development Platform

\section{INTRODUCTION}

Today, the use of a heart-lung machine (HLM) is a safe and routinely used technique in cardiac surgery. Currently the HLM is operated by perfusionists in cooperation with anesthesiologists and heart surgeons in the operating room. Patients suffering from cardiogenic shock would benefit with an early application of a portable extra-corporal circulatory support system (ECSS) preventing multi organ failure. This however requires the presence and constant supervision of the patient by trained personal at the emergency site. With the automatization of a portable ECSS optimal perfusion may be achieved with minimal workload for the human operator.

The focus of this research is the development of an adaptive and robust control system that regulates perfusion based on online data of the patient. While the system needs to be highly dynamic, so that it is able to adapt to different situations, it must ensure maximal patient safety at all times.

The "AutoMedic" platform we present is constructed to help engineers together with medical doctors in the design process of an automatized ECSS. In order to control and optimize perfusion, we focus on several parameters from the patient: temperature, oxygen saturation, heart rate, ECG, blood flow and blood pressure. The observed parameters of the ECSS are: pump speed, inlet pressure, outlet pressure and flow. AutoMedic allows the recording of sensor data that may be used as an input for the control loop. Moreover, the recorded data can be used offline together with the platform for simulations and tests of different control units. Preliminary work needs to be conducted to establish a self-contained, robust and interactive control system. First, real data of the previously listed parameters needs to be obtained, representing different scenarios. This is achieved in animal experiments where a pig model is established. Based on this data, a hydraulic model of the circulatory system is build up. Such a model allows the analysis of the system behavior and mutual interaction between the different parameters and possible control variables. Data acquired from the hydraulic model can then be used to create a mathematical model. This is necessary in order to create a rule base for the controller. In the following we describe our approach and methods in detail and give a description of the AutoMedic framework.

\section{Methods}

\section{A. Portable Heart-Lung Machine}

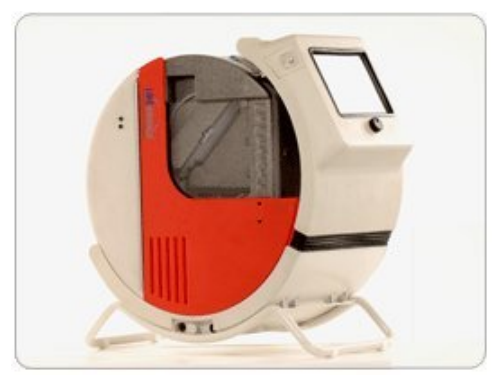

Fig. 1: LifeBridge $B_{2} T$

The LifeBridge $\mathrm{B}_{2} \mathrm{~T}$ System (Fig.1) is a modular extracorporal circulatory emergency support system weighing 17 


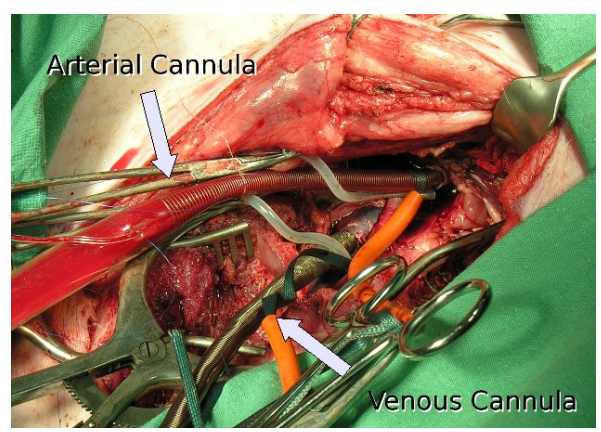

Fig. 2: Arterial and Venous Cannula

$\mathrm{kg}$, characterized by the following features: modular configuration and semi-automatic priming, portability including integrated power supply offering up to two hours of standalone operation with LiPo batteries, and a seven stage air infusion prevention system which can tolerate massive venous ingress of air without jeopardizing patient safety. System parameters can be gathered and recorded in real-time via a serial interface. First clinical experiences with the LifeBridge $\mathrm{B}_{2} \mathrm{~T}$ are described by Krane et al. [1]

\section{B. Animal Experiments}

The experiments are conducted using ordinary pigs of approximately $80 \mathrm{~kg}$. The parameters used in our experiments are the following:

\section{Pig parameters}

- Blood Pressure: disposable pressure transducers together with Millar Mikro-Tip [2] ultra-miniature pressure catheters ( 2 French) that are introduced into the arteries for invasive monitoring.

- Blood Flow: Ultrasonic flow probes from Transonic [3] measure the flow in the Aorta Ascendens and Descendens.

- SpO2: non invasive pulse oximeter from Corscience [4] placed at the nose.

- ECG:4 lead electrocardiogram board from Corscience [4].

- Blood parameters: potassium, $\mathrm{pH}$ and $\mathrm{pCO} 2$ : Terumo blood parameter monitoring system CDI 500 [5].

- Temperature: temperature transducer.

\section{ECSS parameters}

- Inlet, outlet pressures and pressure before oxygenator.

- Flow generated by the centrifugal pump.

- Speed of the centrifugal pump in RPM.
- Gas blender for oxygenator (Mix percentage of oxygen and air, volume).

The Lifbridge $\mathrm{B}_{2} \mathrm{~T}$ is attached to the pig by cannulation of the femoral artery and vein.(Fig.2)

Data Acquisition and Recording The components previously described have different interfaces. A special Data Acquisition Interface (DAI) was created. This connects all the components to a central recording system. For the analog signals a 16 bit data acquisition board was used with a maximum of 16 channels sampling at $200 \mathrm{~Hz}$ each. The other components were connected using a serial interface with their respective drivers. Other applications are able to obtain the different parameters connected to the DAI via a local network. All the signals are recorded in a raw format and through a Monitor application they are displayed during the experiment (see Fig.3).

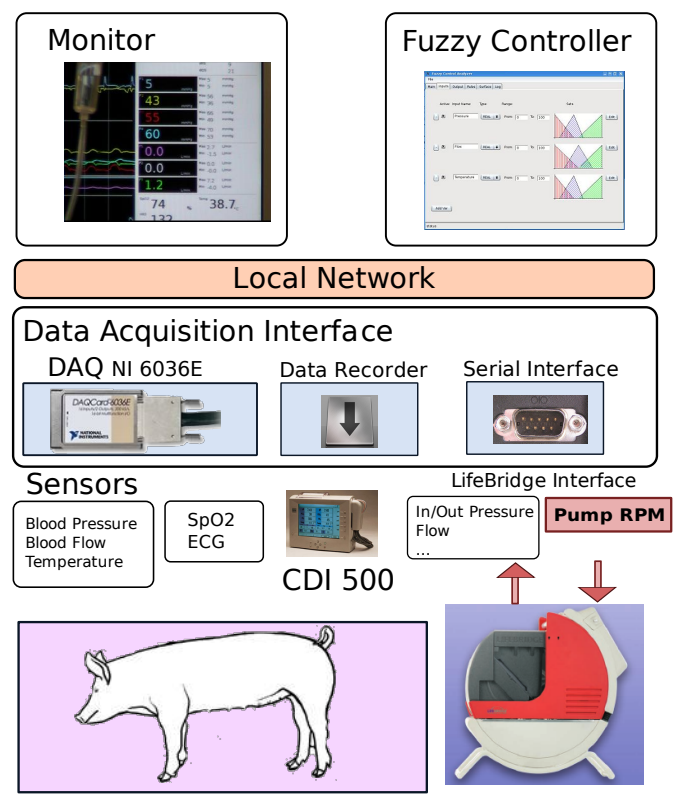

Fig. 3: AutoMedic

Experimental Protocol Throughout animal experiments different tests are conducted in order to simulate different scenarios, following an elaborated protocol. This includes the following:

- Steady perfusion.

- Kinking of tubing .

- Increase of centrifugal pump speed of ECSS. 
- SpO2 Monitoring.

- Lifting of ECSS to different heights.

- Ventricular fibrillation.

- Vibration of the system.

\section{Hydraulic model}

For a hydraulic model several prototypes of previous research were analyzed $[6,7,8,9]$. We choose the socalled "Westerhof model", elaborately described by Sharp and Dharmalingam [9]. It consists of two resistance and one compliance elements in a RCR configuration. The resistance elements are proportional valves from the Burkert company (Germany) with electronically adjustable resistance. A cylindric tank with a hand pump to control air volume serves as a compliance element.

\section{Mathematical Model}

Additional simulations are considered by using a mathematical model of the cardiovascular system. The following models were considered $[10,11]$. We selected the Physbe model available as an existing block in Matlab Symulink. This model separates the circulatory system into three main blocks: the lungs (pulmonary circulation), the heart (coronary circulation), and the rest of the system (systemic circulation). From these blocks subsystems are modeled: the right heart, left heart, vena cava and aorta for the coronary circulatory system and arms head trunk and legs for the systemic circulatory system [11]. Furthermore a mathematical model of the LifeBridge $B_{2} T$ was implemented with the use of experimental data. The connection between both models is done by connecting the vena cava and the aorta of the Physbe model to a venous and arterial cannula and to the input and output respectively of the ECSS model. (Fig. 4).

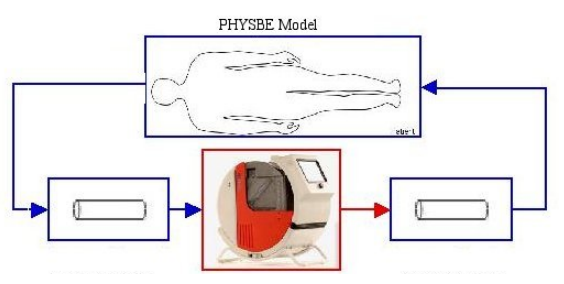

Fig. 4: Mathematical Model

\section{E. Fuzzy Control}

As a control mechanism a fuzzy-logic controller was selected. Several fuzzy control development systems were analyzed to be used for the design of the controller: the Free Fuzzy Logic Library [12], XFuzzy [13] and the Fuzzy Logic
Toolbox from Matlab [14]. A Fuzzy Control Unit was implemented and is connected to the DAI.

\section{RESULTS}

\section{A. Animal Experiments}

Four animal experiments were conducted with approx. 80 kilogram pigs. The mentioned parameters were successfully recorded using the AutoMedic platform (Fig.5). The protocol described was executed and each step was analyzed in how the different parameters were affected.

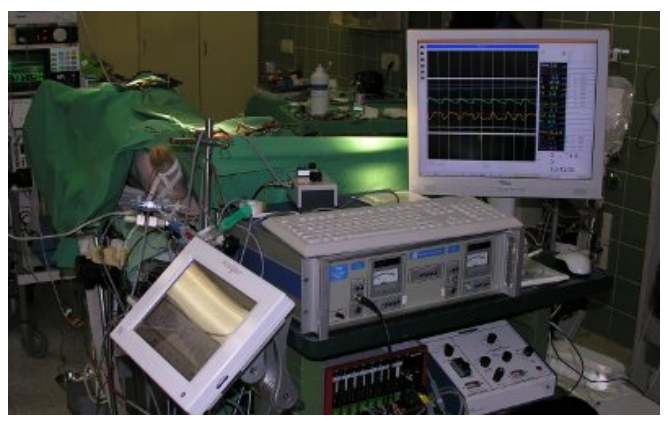

Fig. 5: Animal Experiment Setup

As an example figure 6 shows experiment data. PIn and POut represents the pressures meassured at the input and output tubing of the ECSS. POx is the pressure right before the Oxigenator inside the ECSS. The blood flow was measured at the output. The pump speed was increased gradually until reaching a flow of aprox. $5 \mathrm{~L} / \mathrm{min}$. With this we are able to test the reaction of pressures and flow with respect to the RPM of the pump. From the experimental we can observe an almost linear behavior between the pump speed and the blood flow.

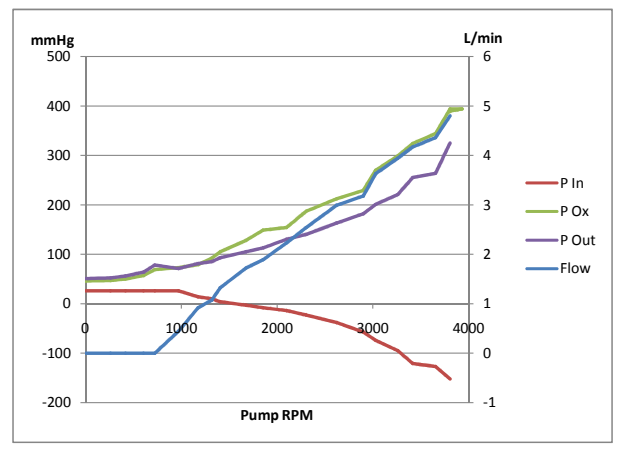

Fig. 6: Experimental Data

The data obtained from the animal experiments was used to setup the hydraulic model and the mathematical model. 
The input and output resistance were set to represent the animal characteristics. The response of both models were compared with the experimental data giving a good approximation.

\section{DISCUSSION}

The animal experiments are essential for this project since they give the possibility to obtain data which are closest to a real situation. To assure this, special attention was given to the capturing of the different parameters. Calibration and synchronization of all devices was indispensable. The data of the different experiments are used as input parameters of the hydraulic and mathematical model.

The hydraulic model plays an important role in making sure that all of the different components are functioning correctly before animal experiments take place. Furthermore, the influence of different variables of both the ECSS and the patient can be analyzed in a reproducible setup. Up until now, many models of the human circulatory system have been established. The complexity of these models however makes a difficult implementation. The here used "Westerhof model" on the other hand has few components and still give a close representation of the cardiovascular system.

In the mathematical model parameters of the cardiovascular system may be changed to simulate different scenarios of patients with specific characteristics. The reaction of the controller may then be analyzed to further improve its design. In this specific model it is possible to simulate the interaction of a beating heart with the ECSS. Fuzzy Logic seems to be an appropriate approach for this project, since it allows to represent experts' knowledge by linguistic rules. Like this, surgeons and perfusionists are able to contribute their experience in the controller design without having to know anything about the technical realization. Besides, fuzzy terms as "more or less" or "a bit" are representable in a standardized way.

\section{CONCLUSiON}

The early use of a portable ECSS in patients with cardiogenic shock could be a possible treatment to improve the survival rates of these patients. In emergency situations the ECSS should be able to work automatically without the need of specialized personnel. This may only be possible with safety procedures that will not put the patient at risk and with a robust system capable of adapting to various patients. With the AutoMedic development platform different approaches are established for a thorough test of the system. From the animal experiments we are able to extract data that represent real-life situations. The gathered data is then applied as input parameters for the hydraulic and mathematical model. These two models are used for the design of the fuzzy controller where intensive evaluation of the system is possible by creating different setups while improving the rules applied for automatization. As a medical application extensive evaluation will be needed to test the reliability of the system. Since different reactions may occur from one patient to another it will be essential to gather real life data in the next future. As a further step more animal experiments will be conducted in which the designed controller will be compared with the specialized perfusionist. The AutoMedic platform gives in essence the required tools to enable the full automatization of the ECSS for the use in emergency situations.

\section{ACKNOWLEDGMENTS}

This work has been supported by an unrestricted educational grant from Bayerische Forschungsstiftung.

\section{REFERENCES}

1. Krane M Schreiber U Mendoza Garcia A Voss B. Badiu C.C.Lange R.Bauernschmitt R.. First experience with a new portable cardiopulmonary bypass system - LIFEBRIDGE B2T with percutaneous femoral cannulation Comp. In Cardiology. 2008:269-272.

2. Millar Instruments at http://www.millarinstruments.com

3. Transonic at http://www.transonic.com

4. Corscience at http://www.corscience.de

5. Terumo at http://www.terumo-europe.com

6 . WJ. Kolff. Mock circulation to test pumps designed for permanent replacement of damaged hearts. Cleveland Clin Quart. 1959;26:223226.

7. Jr. F. M. Donovan. Design of a Hydraulic Analog of the Circulatory System for Evaluating Artificial Hearts Biomater Med Devices Artif Organs. 1975;3(4):439-449.

8. Rosenberg G Landis DL Pierce WS. Design and Evaluation of the. Pennsylvania State University Mock Circulatory System ASAIO. 1981;4(2):41-49.

9. Sharp MK, Dharmalingam RK. Development of a hydraulic model of the human systemic circulation ASAIO. 1999;45:535-540.

10. Bauernschmitt R Naujokat $E$ Vahl CF black head A Kiencke U Hagl S. Mathematical modeling of extracorporeal circulation: Simulation of different perfusion regimens Perfusion Perfusion. 1999;14:321-330.

11. McLeod John. Physbe a physiological simulator. Simulation. 1966;7(6):324-329.

12. Free Fuzzy Logic Library at http://ffll.sourceforge.net/

13. XFuzzy 3.0 at http://www.imse.cnm.es/Xfuzzy/

14. Fuzzy Logic Toolbox at http://www.mathworks.com/products/fuzzylogic/ 